
\title{
is Research Suare \\ Exploration for olive fruit fly parasitoids across Africa - regional distributions and dominance of co-evolved parasitoids
}

\author{
Xingeng Wang \\ Agricultural Research Service \\ Vaughn Walton \\ Oregon State University, United States \\ Kim Hoelmer \\ Agricultural Research Service \\ Charles Pickett \\ California Department of Food and Agriculture \\ Arnaud Blanchet \\ USDA European Biological Control Laboratory \\ Robert Straser \\ University of California, Berkeley \\ Alan Kirk \\ USDA European Biological Control Laboratory \\ Kent Daane ( $\boldsymbol{\nabla}$ kmdaane@berkeley.edu ) \\ University of California, Berkeley
}

\section{Research Article}

Keywords: olive fruit fly, parasitoids

Posted Date: December 18th, 2020

DOI: https://doi.org/10.21203/rs.3.rs-126209/v1

License: (a) (i) This work is licensed under a Creative Commons Attribution 4.0 International License. Read Full License

Version of Record: A version of this preprint was published at Scientific Reports on March 17th, 2021. See the published version at https://doi.org/10.1038/s41598-021-85253-y. 


\section{Abstract}

The olive fruit fly, Bactrocera oleae, has been a key pest of olives in invaded regions Europe and North America. We conducted the largest modern exploration for the fly's co-evolved parasitoids across Sub-Saharan Africa (Kenya, Namibia, and South Africa) and some of the fly's expanded regions (Canary Islands, China, India, Morocco, Pakistan, Réunion Island and Tunisia). From Sub-Saharan regions, four native braconids, Psytallia lounsburyi, P. humilis, Utetes africanus and Bracon celerwere collected. Principal Component Analysis showed that the regional dominance of these parasitoid species was related to climate niches, with $P$. lounsburyi the dominant species in the more tropical areas of Kenya, $P$. humilis dominant in the hot semi-arid areas of Namibia and $U$. africanus prevalent in Mediterranean climates of South Africa. Psytallia concolor was found in the Canary Islands, Moroccan and Tunisian, and the Afrotropical braconid Diachasmimorpha longicaudata in Réunion Island. In South Africa, seasonal monitoring of $B$. oleae showed consistently low infestation in unripe or ripe fruits. Multivariate analyses suggest that fruit maturity, seasonal climates and interspecific interactions shape the local parasitoid diversity that effectively regulates fly populations at low levels. The results are discussed with regard to ecological adaptations of co-evolved parasitoids, and how their adaptations impact biocontrol.

\section{Introduction}

Exotic insect pests often thrive in their invaded regions due to the absence of co-evolved natural enemies and lack of effective indigenous natural enemies ${ }^{1,2}$. Classical biological control (CBC) by the introduction of coevolved natural enemies from the exotic pest's native range is an attempt to restore the pest-natural enemy balance after an invasion event ${ }^{3-5}$. Economic returns on successful programs are overwhelmingly positive ${ }^{6}$, but $\mathrm{CBC}$ programs require proper steps to be successfully implemented and to reduce inconsequential natural enemy releases or negative nontarget impacts 7,8 . For herbivorous invaders, this requires a fundamental understanding of the natural enemy impact in its native range, biology and host specificity, as well as potential tri-trophic interactions that develop from a high degree of co-adaptation between plant-herbivore-carnivore and the impact of habitat and environment on the selected natural enemy ${ }^{7}$. One aspect is matching the climatic niches occupied by the natural enemies in the native range to the invaded range ${ }^{9}$. Climate matching has been particularly important in fruit fly biological control programs ${ }^{10,11}$. Hymenopteran parasitoids from the braconid subfamily Opiinae have been used worldwide in CBC programs to control fruit-infesting Tephritidae ${ }^{12-}$ 16. The vast majority of utilized braconid parasitoids are koinobiont endoparasitoids that oviposit in the host egg or larval stage and emerge from host pupae ${ }^{17}$. Therefore, the adult female parasitoid must first locate and attack the concealed immature stages of host fly inside the fruit, then bypass the host immune response, and successfully develop. For these reasons, opiine parasitoids are generally highly co-evolved with their associated host species.

The olive fruit fly, Bactrocera oleae (Rossi) (Diptera: Tephritidae) has been a key pest of cultivated olives throughout the Mediterranean Basin and North America, largely due to a lack of effective natural enemies in these invaded regions ${ }^{18}$. The fly larvae feed exclusively in olives ${ }^{19}$, both cultivated olives, Olea europaea ssp. europaea (Wall ex G. Don), and wild olives, of which various subspecies, occur widely in parts of Africa, southern Europe and southwestern Asia ${ }^{20,21}$. The fly's current range extends throughout the Mediterranean 
Basin, northern and Sub-Saharan Africa, southwestern Asia (parts of India, Pakistan and China), and North America (California and Mexico) ${ }^{18,21}$. Population structure and genetic analyses suggest that $B$. oleae is native to Sub-Saharan Africa and then likely moved into North Africa and later the Mediterranean Basin, then proceeded westward through Europe and eventually North America ${ }^{22-24}$. The close association of the fly and olives suggests the existence of highly co-evolved parasitoids associated with $B$. oleae. In fact, indigenous parasitoids attacking $B$. oleae in the Mediterranean Basin are generalist chalcidoids such as Eurytoma martellii Dom. (Eurytomidae), Eupelmus urozonus Dalm. (Eupelmidae), Pnigalio agraules Walk. (Eulophidae) and Cyrtoptyx latipes Rond. (Pteromalidae) ${ }^{25,26}$. Similarly, the indigenous parasitoid attacking $B$. oleae in California, Pteromalus nr. sp. myopitae (Pteromalidae), is also a generalist ${ }^{27}$. These species are idiobiont ectoparasitoids, placing their eggs on the host surface and may not need to overcome internal host defenses, thus are polyphagous, attacking even unrelated insect hosts. While present, these generalist parasitoids do not provide effective $B$. oleae control.

The first major attempt to introduce coevolved parasitoids to suppress $B$. oleae populations dates to the early 1900 s with the exploration for natural enemies in Africa to be released in Italy by Filippo Silvestri ${ }^{28}$. The early explorations discovered and described several braconid species collected from $B$. oleae including Psytallia concolor (Szépligeti), P. lounsburyi (Silvestri), Utetes africanus (Szépligeti) and Bracon celer Szépligeti collected in South Africa, Kenya and Ethiopia reviewed in $18,29,30$. However, none of these parasitoids were successfully cultured by Silvestri and only small numbers of some of these parasitoids were released in Italy without subsequent establishment ${ }^{31}$. Only $P$. concolor, obtained from Tunisia, was repeatedly introduced since the early 1900s and extensively released in the Mediterranean Basin, but this species has established only in some southern regions and does not provide effective control ${ }^{32,33}$. Still, there has been continued interest in massrearing and releasing $P$. concolor and/or $P$. lounsburyi to improve sustainable fly management in Europe ${ }^{34-36}$.

The invasion and widespread establishment of $B$. oleae in California and northwestern Mexico initiated renewed interest in the classical biological control of this pest ${ }^{37}$. Modern exploration for effective natural enemies was designed to include the fly's likely native ranges in Sub-Saharan Africa (Kenya, South Africa and Namibia) and some expanded regions in Africa (Canary Islands, Morocco, Tunisia and Réunion Island). Here we present a comprehensive analysis of the regional distribution, diversity, and dominance of braconid $B$. oleae parasitoids in these seven African regions and examine how the regional dominance might be related to regional climatic variables. Furthermore, we analyzed the seasonal dynamics of $B$. oleae and its co-evolved parasitoids in South Africa, one of the native regions with a high diversity of host-specific parasitoids, and examined how some biotic and abiotic factors might have shaped the local diversity of the parasitoid complex that effectively regulates $B$. oleae populations at low levels. This framework may provide new insights into the nature of climate niches of different parasitoid species and their associated tri-trophic interactions in guiding the design of ongoing classical biological control programs in California and the Mediterranean Basin.

\section{Results}

Parasitoid regional distribution and diversity. Surveys from 110 sites of wild olives, 0 . e. nr. ssp. cuspidata, in seven African regions yielded a total of 443,308 olive berries (Fig. 1), of which 72,453 fly pupae, 27,848 adult $B$. oleae and 22,576 adult braconid parasitoids were obtained (Table 1). Two closely related African Bactrocera 
species, B. biguttula (Bezzi) (1.2\%) and B. munroi White (2.6\%) were also recovered, but in low numbers and with $B$. biguttula found only in South Africa and B. munroi only in Kenya (Table 1). Five Opiinae braconid wasps were recovered: $P$. concolor, P. lounsburyi, P. humilis (Szépligeti), U. africanus and Diachasmimorpha longicaudata (Ashmead); one Braconinae braconid wasp, B. celer, was also recovered. Psytallia concolor was the only species found in the Canary Islands, Tunisia and Morocco, whereas $D$. Iongicaudata was the only species recovered in the Réunion Island (Fig. 1). The other four species were found in Namibia and South Africa and three of them (except $P$. humilis) were found in Kenya, with $P$. lounsburyi, $P$. humilis and $U$. africanus being the predominant parasitoid species in Kenya, Namibia and South Africa, respectively (Fig. 1).

Table 1. Numbers of wild olive fruit collected, fly pupae, adult flies of $B$. oleae, B. biguttula and $B$. munroi, and braconid parasitoids obtained from the collections in different regions during 2002 to 2011

\begin{tabular}{lrrrrrrr}
\hline Region & $\begin{array}{c}\text { Year } \\
\text { (no. of } \\
\text { Sites) }\end{array}$ & Fruit & $\begin{array}{c}\text { Fly } \\
\text { pupae }\end{array}$ & $\begin{array}{c}B . \\
\text { oleae }\end{array}$ & $\begin{array}{c}\text { B. } \\
\text { biguttula }\end{array}$ & $\begin{array}{c}\text { B. } \\
\text { munroi }\end{array}$ & Braconids \\
\hline Tunisia & $2000(3)$ & 912 & 710 & 602 & 0 & 0 & 108 \\
Morocco & $2004(7)$ & 12044 & 487 & 316 & 0 & 0 & 41 \\
Réunion & $2004(8)$ & 16666 & 1786 & 700 & 0 & 0 & 114 \\
Namibia & $2004(3)$ & 5595 & 756 & 413 & 0 & 0 & 188 \\
& $2005(1)$ & 700 & 35 & 17 & 0 & 0 & 1 \\
& $2007(3)$ & 15862 & 2090 & 1034 & 0 & 0 & 601 \\
& $2008(6)$ & 11583 & 5440 & 1402 & 0 & 0 & 1700 \\
& $2009(4)$ & 7070 & 1539 & 884 & 0 & 0 & 218 \\
Kenya & $2011(2)$ & 13639 & 11 & 6 & 0 & 0 & 1 \\
& $2002(1)$ & 45762 & 10364 & 3309 & 0 & 0 & 5039 \\
& $2003(2)$ & 71680 & 16291 & 5177 & 0 & 0 & 8432 \\
& $2004(1)$ & 440 & 100 & 38 & 0 & 0 & 21 \\
& $2005(5)$ & 41008 & 9321 & 4020 & 0 & 335 & 2125 \\
& $2006(1)$ & 29040 & 6600 & 1834 & 0 & 21 & 264 \\
South & $2007(3)$ & 28864 & 6560 & 2277 & 0 & 16 & 2402 \\
Africa & $2008(2)$ & 13200 & 3000 & 1314 & 0 & 108 & 668 \\
& $2003(29)$ & 74579 & 2323 & 1095 & 0 & 0 & 244 \\
& $2004(16)$ & 25885 & 1095 & 400 & 0 & 0 & 157 \\
& $2005(6)$ & 22470 & 800 & 388 & 23 & 0 & 125 \\
\hline
\end{tabular}

Total parasitism was higher in the three Sub-Saharan regions than in other regions $\left(F_{6,84}=4.5, p<0.001\right)$ (Fig. $3 A)$. Diversity of the braconid parasitoid complex was similar among the three Sub-Saharan regions $\left(F_{6,66}=4.5\right.$, $p<0.001)$ (Fig. 3B). The percentage of female wasps was $64.4 \pm 10.3 \%(\mathrm{n}=11$, number of sites) for $P$. concolor, $50.3 \pm 4.0 \%(\mathrm{n}=42)$ for $P$. lounsburyi, $65.4 \pm 6.2 \%(\mathrm{n}=28)$ for $P$. humilis, $60.3 \pm 3.5 \%(\mathrm{n}=50)$ for $U$. africanus, $43.4 \pm 8.6 \%(n=9)$ for $D$. $n$ r. sp. longicaudata and $24.8 \pm 4.8 \%(n=35)$ for $B$. celer. The sex ratios were similar among the five opiine parasitoids but was higher than the braconine parasitoid $\left(F_{5,170}=7.9, p<\right.$ $0.001)$. 
PCA revealed two significant components that jointly explained $79.1 \%$ of the variance in the regional climatic variables (eigenvalues: component $1=3.76,46.9 \%$ of variance; component $2=2.58,32.2 \%$ of the variance) (Fig. 4). Despite the overlap of a few sites, the explored regions represented clearly different climate types. The climates in the Canary Islands, Morocco and Namibia were similar and are characterized by high temperatures and low precipitation. However, the climates in Kenya were related positively to the precipitation but negatively to the maximum temperature of the warmest month with South Africa falling between these two climate types. The Réunion climates were highly correlated to precipitation. The regional dominance of the parasitoid species was reflected in the PCA ordination (eigenvalues: component $1=5.62,51.1 \%$ of variance; component $2=2.17$, $19.7 \%$ of the variance) (Fig. 5). Sites in Kenya were assigned on the left while sites in Namibia were assigned on the right, and those in South Africa were in the middle. There was a positive relationship between the annual mean temperature or maximum temperature of the warmest month and the relative abundance of $P$. humilis, however this relationship was negative for $P$. lounsburyi. The relative abundance of $U$. africanus was negatively correlated with the minimum temperature of the coldest month and strongly dependent on the precipitation during the wettest quarter.

Seasonal host-parasitoid dynamics. Independent of the regional surveys, more localized surveys were conducted at 24 sites of wild olives, O. e. nr. ssp. cuspidata, at the Western Cape Province that yielded a total of 252,603 unripe and 139,872 ripe wild olive fruit were collected (Table 2). Ripe fruit (pulp thickness $=1.93 \pm 0.06$, $\mathrm{n}=43$ ) were significantly larger than unripe fruit (pulp thickness $=0.94 \pm 0.04, \mathrm{n}=32)\left(F_{1,73}=169.4, p<0.001\right)$. Mean monthly host density (or fruit infestation rate) on the unripe and ripe fruit were $0.5-11.7 \%$ (mean $=4.5 \pm$ $0.7 \%$ ) and $4.6-41.2 \%$, (mean $=14.8 \pm 2.0 \%$ ), respectively (Fig. 6A). Host density increased with fruit maturity and was affected by the interaction between fruit maturity and seasonal temperature (Table 3). Most emerged flies were $B$. oleae. Only $0.87 \pm 0.39 \%$ and $1.90 \pm 0.66 \%(n=24)$ of the emerged flies from the unripe and ripe fruit were B. biguttula. Mean combined parasitism of $B$. oleae and B. biguttula were $28.6 \pm 2.8 \%$ and $25.4 \pm$ $2.6 \%$ on the unripe and ripe fruit, respectively. The parasitism was not affected by fruit maturity but rather negatively related to mean temperature (Table 3), decreasing only during mid-summer months (Fig. 6B).

Table 2. Numbers of wild olives collected and fly pupae, adult flies of $B$. oleae (Bo) and $B$. biguttula $(\mathrm{Bb})$ and braconid parasitoids emerged from collected unripe and ripe fruit in 26 different sites in the Western Cape, South Africa from October 2004 to February 2007 


\begin{tabular}{|c|c|c|c|c|c|c|c|c|c|c|}
\hline \multirow{2}{*}{$\begin{array}{l}\text { Collection } \\
\text { site } \\
\text { (city, code) }\end{array}$} & \multicolumn{5}{|c|}{ Collections of unripe fruit } & \multicolumn{5}{|c|}{ Collections of ripe fruit } \\
\hline & Fruit & Pupae & Bo & $\mathrm{Bb}$ & Parasitoids & Fruit & Pupae & Bo & $\mathrm{Bb}$ & Parasitoids \\
\hline $\begin{array}{l}\text { Cape Town } \\
1\end{array}$ & 552 & 63 & 29 & 0 & 5 & 208 & 13 & 5 & 0 & 6 \\
\hline $\begin{array}{l}\text { Stellenbosch } \\
2\end{array}$ & 14338 & 3428 & 879 & 11 & 402 & 14943 & 523 & 239 & 0 & 84 \\
\hline Stellenbosch & 6927 & 949 & 275 & 17 & 119 & 9786 & 79 & 53 & 0 & 9 \\
\hline Stellenbosch & 8002 & 1448 & 533 & 72 & 228 & 19142 & 355 & 140 & 8 & 101 \\
\hline $\begin{array}{l}\text { Stellenbosch } \\
5\end{array}$ & 4795 & 1290 & 536 & 48 & 216 & 8825 & 287 & 111 & 9 & 55 \\
\hline $\begin{array}{l}\text { Stellenbosch } \\
6\end{array}$ & 11162 & 1723 & 706 & 37 & 289 & 17026 & 290 & 158 & 3 & 54 \\
\hline $\begin{array}{l}\text { Stellenbosch } \\
7\end{array}$ & 15833 & 1176 & 453 & 8 & 192 & 32580 & 382 & 168 & 3 & 66 \\
\hline $\begin{array}{l}\text { Stellenbosch } \\
8\end{array}$ & 919 & 78 & 67 & 0 & 5 & 891 & 51 & 35 & 0 & 3 \\
\hline $\begin{array}{l}\text { Stellenbosch } \\
9\end{array}$ & 3983 & 5 & 1 & 0 & 0 & 438 & 9 & 7 & 0 & 0 \\
\hline $\begin{array}{l}\text { Stellenbosch } \\
10\end{array}$ & 2073 & 50 & 27 & 0 & 11 & 4795 & 56 & 24 & 0 & 16 \\
\hline Bonnievale 1 & 5438 & 895 & 338 & 26 & 256 & 5965 & 248 & 85 & 3 & 83 \\
\hline Paarl 1 & 14319 & 4530 & 2102 & 48 & 892 & 28199 & 1151 & 632 & 5 & 259 \\
\hline Paarl 2 & 14420 & 3425 & 1626 & 13 & 709 & 31438 & 1387 & 613 & 0 & 377 \\
\hline Paarl 2 & 8706 & 1265 & 631 & 1 & 118 & 13591 & 1031 & 612 & 1 & 119 \\
\hline Paarl 4 & 5665 & 694 & 360 & 0 & 154 & 16166 & 549 & 267 & 0 & 154 \\
\hline Paarl 5 & 4342 & 288 & 157 & 2 & 57 & 9306 & 249 & 124 & 0 & 87 \\
\hline Paarl 6 & 7408 & 2040 & 856 & 2 & 350 & 11062 & 1113 & 608 & 0 & 95 \\
\hline Wellington 1 & 7091 & 676 & 311 & 0 & 167 & 9923 & 198 & 94 & 0 & 58 \\
\hline Citrusdal 1 & 1323 & 124 & 54 & 0 & 13 & 2621 & 123 & 70 & 0 & 18 \\
\hline Citrusdal 2 & 2722 & 99 & 49 & 0 & 17 & 4595 & 78 & 31 & 0 & 20 \\
\hline Citrusdal 3 & 227 & 90 & 21 & 0 & 1 & 1335 & 193 & 76 & 0 & 26 \\
\hline Citrusdal 4 & 2052 & 114 & 55 & 0 & 28 & 3997 & 116 & 37 & 0 & 15 \\
\hline Citrusdal 5 & 222 & 24 & 4 & 0 & 6 & 515 & 32 & 13 & 0 & 8 \\
\hline Citrusdal 6 & 898 & 130 & 82 & 0 & 7 & 1711 & 230 & 174 & 0 & 8 \\
\hline
\end{tabular}

Table 3. Mixed Models analyzing the effects of fruit maturity (unripe vs. ripe), mean monthly temperature as well as the interactions of these two factors on fruit infestation rate and parasitism in Western Cape, South Africa 


\begin{tabular}{llrrr}
\hline Parameter & Variables & Estimate \pm SE & $t$ & $P$ \\
\hline \% Fruit infested & Fruit maturity (FM) & $0.098 \pm 0.015$ & 6.62 & $<0.001$ \\
& Mean temperature (MT) & $-0.003 \pm 0.003$ & 0.97 & 0.335 \\
& FM $\times$ MT & $-0.008 \pm 0.003$ & 2.24 & 0.029 \\
& & & & \\
Parasitism & Fruit maturity & $-0.039 \pm 0.051$ & -0.77 & 0.446 \\
& Mean temperature & $-0.029 \pm 0.012$ & -2.47 & 0.017 \\
& FM $\times$ MT & $-0.005 \pm 0.012$ & -0.40 & 0.688 \\
\hline
\end{tabular}

All four braconid parasitoids, $P$. lounsburyi, $P$. humilis, $U$. africanus and $B$. celer, were found in both unripe and ripe fruit. Diversity was generally higher in ripe than unripe fruit (Fig. 7A). U. africanus was the predominant parasitoid, followed by $P$. lounsburyi while both $P$. humilis (mean $1.8 \%$ and $2.5 \%$ on the unripe and ripe fruit, respectively) and $B$. celer (mean $0.1 \%$ and $4.9 \%$ on unipe and ripe fruit, respectively) were much less common in both ripe (Fig. 7B) and unripe (Fig. 7C) fruit. GLM analyses showed that diversity was not affected by mean temperature but was positively related to fruit maturity and host density. The relative abundance of $U$. africanus was affected negatively by fruit maturity and the presence of other parasitoid species but was positively related to host density (Table 4). The relative abundance of $P$. lounsburyi was affected only by the presence of other parasitoids (Table 4).

Table 4. Generalized Linear Model testing the effects of (1) fruit maturity (unripe vs. ripe), mean monthly temperature and host density (= \% fruit infested) on diversity of braconid parasitoids, and (2) fruit maturity, mean monthly temperature, host density, and incidence of other braconids on the relative abundance of the dominant braconids $U$. africanus or $P$. lounsburyi in Western Cape, South Africa

\begin{tabular}{|c|c|c|c|c|}
\hline Parameter & Variables & Estimate $\pm \mathrm{SE}$ & $c^{2}$ & $P$ \\
\hline \multirow[t]{3}{*}{ Diversity } & Fruit maturity & $0.68 \pm 0.23$ & 9.70 & $0.002 *$ \\
\hline & Mean temperature & $0.03 \pm 0.02$ & 1.92 & 0.166 \\
\hline & Host density & $1.21 \pm 0.43$ & 7.05 & $0.008^{*}$ \\
\hline \multirow[t]{6}{*}{$\%$ U. africanus } & Fruit maturity & $-0.64 \pm 0.30$ & 4.71 & $0.030 *$ \\
\hline & Mean temperature & $-0.04 \pm 0.03$ & 1.49 & 0.221 \\
\hline & Host density & $2.17 \pm 0.81$ & 7.91 & $0.005^{*}$ \\
\hline & Presence of $P$. lounsburyi & $-2.63 \pm 0.32$ & 90.08 & $<0.001 *$ \\
\hline & Presence of $P$. humilis & $-1.64 \pm 0.40$ & 16.88 & $<0.001 *$ \\
\hline & Presence of $B$. celer & $-1.97 \pm 0.53$ & 15.11 & $<0.001 *$ \\
\hline \multirow[t]{6}{*}{$\%$ P. lounsburyi } & Fruit maturity & $0.14 \pm 0.42$ & 0.12 & 0.727 \\
\hline & Mean temperature & $0.05 \pm 0.04$ & 1.17 & 0.279 \\
\hline & Host density & $0.53 \pm 0.92$ & 0.33 & 0.564 \\
\hline & Presence of $U$. africanus & $-5.79 \pm 0.57$ & 172.57 & $<0.001 *$ \\
\hline & Presence of $P$. humilis & $-5.59 \pm 2.39$ & 13.90 & $<0.001 *$ \\
\hline & Presence of B. celer & $-5.74 \pm 1.89$ & 17.22 & $<0.001 *$ \\
\hline
\end{tabular}




\section{Discussion}

We conducted the largest modern exploration for olive fruit fly parasitoids in Africa. Our surveys reveal remarkable differences in distribution, diversity and dominance of braconid parasitoid guilds from wild olives across the African continent. The sub-Saharan regions of Namibia, South Africa and Kenya maintained the highest diversity of braconid $B$. oleae parasitoid species, supporting the argument of a Sub-Saharan origin of $B$. oleae ${ }^{22-24}$. We found only one native braconid parasitoid (P. concolor) in northern Africa, despite climates in the sampled regions being similar to that of Namibia. We recovered only the introduced parasitoid, $D$. Iongicaudata, in Réunion Island where the native Afrotropical species Diachasmimorpha fullawayi (Silvestri) was reported from other tephritid fruit flies ${ }^{38}$. No braconid parasitoids are reported to occur naturally in Europe's Mediterranean Basin ${ }^{25,26}$ or California ${ }^{37}$. Outside Africa, surveys were conducted in India, Nepal, Pakistan and China and from these collections another braconid parasitoid, Psytallia ponerophaga (Silvestri), was reared from B. oleae in Pakistan and D. longicaudata was recovered in China 21,39 .

All five parasitoids reared from $B$. oleae are larval parasitoids and four of them (P. lounsburyi, P. humilis, $U$. africanus and $D$. longicaudata) are koinobiont endoparasitic opiine wasps; $B$. celer is an idiobiont ectoparasitic braconine wasp 40-42. Among tephritid fruit fly parasitoids, only a few are braconine parasitoids and nearly all of these are idiobiont ectoparasitoids of the larval flies ${ }^{17}$. No egg parasitoids of $B$. oleae were found in the current survey reported herein, or in previous surveys $12,28,30,31,43$, although one generalist egg parasitoid, Fopius arisanus (Sonan) (Hymenoptera: Braconidae) was able to attack and develop from B. oleae under quarantine conditions ${ }^{44}$. In our collections, parasitoids were obtained from pupae collected after exiting fruit or by rearing adults from infested fruit. It is likely that parasitoids that locate and attack hosts in the soil after larvae drop from fruit, or following pupation, have been underrepresented ${ }^{43}$. Some pupal parasitoids such as Pachycrepoideus vindemiae Rondani (Hymenoptera: Pteromalidae) were known to attack B. oleae ${ }^{28}$. Other chalcidoid parasitoid species were reported previously from Africa attacking fruit flies, but they are considered to be generalists and would not be recommended for introduction for biological control e.g., ${ }^{45,46}$. The other two closely related fly species recovered, B. biguttula in South Africa and B. munroi in Kenya, were also collected from wild olives. The collected parasitoids may also attack these two fly hosts, but $B$. oleae is thought to be their major host species as the number of the other two fly species were extremely low in South Africa and Kenya and not recovered in Namibia during our collections.

Four parasitoid species, $P$. lounsburyi, $P$. humilis, $U$. africanus and $B$. celer, were sympatric in the sub-Saharan regions surveyed. However, their dominance varied among regions with different climate types, as determined by PCA. In central Kenya where $P$. lounsburyi was the dominant species, the climate is characterized by mild tropical weather with relatively limited fluctuations in temperature extremes but ample precipitation during the rain months. In contrast, in Namibia where $P$. humilis was the dominant species the climate is typically hot and dry during summer and cold and humid during the winter. Indeed, laboratory studies confirmed that $P$. humilis was more heat-tolerant, yet less cold tolerant, than $P$. lounsburyi ${ }^{47,48}$, which may impact their establishment in 
regions with either hotter summers or colder winters. Although little is known about $U$. africanus' temperature tolerance, the current surveys showed $U$. africanus was more abundant in the Mediterranean-like climates. Many other biotic and abiotic factors could also affect the distribution of these parasitoids. Rainfall patterns would strongly influence the seasonal occurrence and abundance of fruit availability, and consequently the abundance of flies and their parasitoids. In drier habitats, the fruit is likely to be small and ripen slowly, offering little food for fly larvae. Annual precipitation was consistently highest in Kenya, as were olive fly populations and their parasitoids (Table 1). Interspecific competition may occur and coexistence between these species is likely facilitated by niche segregation through differentiation in biological or ecological traits. As shown in South Africa, $U$. africanus was more dominant on small and unripe fruit whereas $P$. lounsbury $i$ was more dominant on ripe fruit. Large ripe fruit may limit the access of $U$. africanus, which has the shortest ovipositor among all five larval parasitoids ${ }^{10}$, but other parasitoids such as $P$. lounsburyi fill the niches. If interspecific competition shapes the parasitoid guilds, it likely would show a similar dominance across different regions. Thus, adaptation to abiotic conditions is likely a major force underpinning diversification and dominance of these species.

All five braconid parasitoids have been imported and evaluated in classical biological control of $B$. oleae in California ${ }^{49}$. In addition, $D$. longicaudata was also found to readily attacks $B$. oleae ${ }^{10,40,50}$, but it is a generalist parasitoid of tephritids ${ }^{38}$. Among the co-evolved African braconid parasitoids, the relatively shorter length of $U$. africanus ovipositors match with lower pulp thickness of wild olives. This parasitoid is ineffective on cultivated olives that has higher pulp thickness through breeding programs. This thicker pulp allows $B$. oleae fly larvae to move deeper into the olive pulp to escape attack from larval parasitoids that have short ovipositors ${ }^{10,50}$. Bracon celer is able to attack the Cape ivy fly, Parafreutreta regalis Munro (Tephritidae: Tephritinae), which itself was introduced from South Africa into California for the control of the invasive Cape Ivy weeds ${ }^{40,51}$. Psytallia concolor is also a common parasitoid of the Mediterranean fruit fly, Ceratitis capitata (Wiedemann) (Diptera: Tephritidae) in eastern Africa ${ }^{12,38,52}$. Although the current surveys did not find it on $B$. oleae in Kenya, it has been previously collected from coffee-infesting C. capitata in other parts of Kenya 52 . Genetic analysis showed clear separation of the North African populations from the Sub-Saharan populations and thereafter referred the Sub-Saharan $P$. concolor populations (often described as $P$. cf. concolor ${ }^{52,53}$ as $P$. humilis ${ }^{54,55}$ ). $P$. lounsburyi has been reared only from $B$. oleae ${ }^{38,52,56,57}$ and is the most host-specialized parasitoid among all parasitoid candidates ${ }^{58}$. In the Mediterranean Basin, $P$. concolor is the only parasitoid that has been extensively studied e.g., ${ }^{59}$ and widely released with partial establishment in the southern regions 60 .

In California, two $P$. humilis populations, originated from $B$. oleae on wild olives in Namibia and $C$. capitata on coffee in Kenya, were released without subsequent establishment ${ }^{37,61-63}$. However, two populations of $P$. lounsburyi, originating from Kenya and South Africa, were released and successfully stablished along the California coastal regions ${ }^{37}$. Low winter survival may contribute to the failure of establishment of $P$. concolor in northern Mediterranean Basin ${ }^{60}$ and $P$. humilis in California 47,64 . For this reason, $P$. ponerophaga from Pakistan is being considered for release in California as it was found to have higher rates of low temperature survival than $P$. humilis ${ }^{65}$. Other factors such as availability of olive fruit for the host-specific $B$. oleae and alternative hosts could also restrict the establishment of its specialized parasitoids in introduced regions. In 
South Africa, wild olives are sufficiently available all year, and alternative hosts may also help parasitoid populations to survive periods when local $B$. oleae populations are sparse 29,52 .

The current study showed that fruit maturity, seasonal climates and interspecific competition likely shape seasonal host-parasitoid dynamics in South Africa. Collectively, the co-adaptation of parasitoids and hosts has resulted in balanced population densities in its native range. Fruit infestation rate was generally less than $15 \%$. In contrast, untreated olives in California can reach $100 \%$ infestation ${ }^{66}$. Although olive fruit fly larvae are not tolerated in fruit used for canning, 10-30\% infested fruit can be tolerated in olives that are pressed for oil in California. The recent successful establishment of $P$. lounsburyi in California should evoke further investigation into the use of the species for classical biological control of $B$. oleae in other climatically similar regions $29,43,49$. The advances of modern rearing techniques for these exotic parasitoid species and their tephritid hosts may further facilitate the use of classical and augmentative biological control of $B$. oleae ${ }^{35}$. However, identifying the suitable climatic niche of these different parasitoid species and understanding their geographic predictability helps to determine the potential establishment in released habitats in the presence of biotic interactions is paramount for successful biological control of $B$. oleae. Alteration of fruit morphological traits (such as size) through domestication may modify the tri-trophic interactions in agricultural eco-systems, reducing the efficiency of the larval parasitoids with short ovipositors on cultivated olives ${ }^{10,40,50}$. Therefore, understanding both the ecological niche and the co-evolutionary history of the host and parasitoid is fundamentally important for effective classical biological control.

\section{Materials And Methods}

Regional exploration. African collections for B. oleae and its parasitoids were conducted from 2000-2011 in seven regions: parts of Kenya, Namibia, South Africa, the Canary Islands, Tunisia, Morocco and Réunion Island (Fig. 1; for detailed locations of collection sites see supplemental Fig. S1). Each year, fruits of wild olives, 0 . e. nr. ssp. cuspidata, were collected, typically during the fruit ripening season in late summer or fall from various habitats including roadsides, hillsides, along stream banks and in woodland landscapes. Sample size (total number of collected fruit) varied among regions, sites and collection dates depending on the availability of fruit. Kenyan surveys were conducted from 2002-2008 at 15 sites in the forests along the southwestern slopes of Mount Kenya in Central Kenya. These sites were located near both sides of the equator and ranged in elevation from 1918-2557 m. Namibian surveys were conducted from 2004-2011 at 18 sites in the Otjozondjupa Province that ranged in elevation from 1409-1557 m. South African surveys were conducted from 2001-2005 at lower elevations ( $<500 \mathrm{~m}$ ) in provinces of the Western Cape (29 sites), Eastern Cape (10 sites) and Gauteng ( 2 sites). The 2001 and 2002 data are not reported fully herein because parasitoid specimens were not always identified to species. Surveys in northern Tunisia were conducted in 2000 at 3 sites. Surveys in the Canary Islands, Morocco and Réunion Island were conducted in 2004, with 9 sites on the Canary Islands (4 on Tenerife, 2 on Gran Canaria and 3 on La Gomera), 7 sites in the South Province of Morocco and 8 sites on the Réunion Island.

Collected fruits were kept at room temperature $\left(20-23^{\circ} \mathrm{C}\right)$ in collaborating laboratories or hotel rooms near collection sites. The fly larvae often pupate inside unripe fruit but will exit and pupate outside of ripe fruit (typically in the soil underneath the tree in situ). When available, the majority of collected fruit were ripe, this allowed an easier collection of the fly puparia emerging from fruit, although both unripe and ripe fruit were 
collected. Larval ectoparasitoids, such as $B$. celer, emerge directly from fruit, which were held for up to one month for maximum emergence of flies or parasitoids. When possible, the emerged pupae were returned with the collector or sent by cooperators to the ARS European Biological Control Laboratory (EBCL), otherwise the material was held at collaborating laboratories for emergence of flies or parasitoids. All emerged insects were identified to species and gender.

Seasonal host-parasitoid dynamics. To monitor the seasonal dynamics of $B$. oleae and its co-evolved parasitoids, collections of wild olives, O. e. ssp. cuspidata, were conducted from October 2004 to February 2007 at 24 fixed sites in the Western Cape Province, South Africa, near Bonnievale, Cape Town, Citrusdal, Paarl, Stellenbosch and Wellington (Fig. 2). These sites were located within $200 \mathrm{~km}$ of each other and ranged in elevation from 77-823 m. Approximately 900 fruits of wild olive were collected at each site once every 2-4 weeks, depending on the availability of fruit. Collected fruit were processed at Stellenbosch University and sorted by size and condition. Fruit size, or pulp thickness, was assumed to affect some parasitoid species ability to find and oviposit into fly larvae feeding deeper inside the fruit because of their short ovipositors ${ }^{10}$. Fully ripe (black) fruit is generally larger than unripe (green) fruit and fly larvae will feed deeper inside the softer fruit. Therefore, green and black fruit were sorted and assessed separately. Subsamples of unripe and ripe fruit were measured to estimate the pulp thickness of each fruit by inserting an insect pin trough the pulp to the seed three times at randomly selected points on the fruit. The mean depth (pin length minus the exposed portion of the pin) of the three measurements was used to estimate fruit pulp thickness. Collected fruit and emerging puparia were kept at room temperature $\left(20-23^{\circ} \mathrm{C}\right)$ until the emergence of wasps and flies.

Data analysis. The relative abundance of each parasitoid species (i.e. percentage of each parasitoid species emerged), total parasitism by all parasitoids and diversity were estimated for each sample in each site and region. Total parasitism was calculated by dividing the total number of emerged parasitoids by the sum of the number of emerged parasitoids and flies. The Shannon index $(H)$ was used to estimate the diversity:

\section{$H=\sum(p i)|\ln (p i)|$}

where $p i$ is the proportion of each parasitoid species. Sex ratio (\% females) of each parasitoid species was pooled from different regions because initial analyses did not detect significant differences for any parasitoid among different regions. Mean parasitism and diversity among different regions and sex ratio among different parasitoid species were compared using one-way ANOVA. All data were first inspected for normality and error variance for homoscedasticity and all percentage data were logit transformed as needed before analysis.

Principal Component Analysis (PCA) was conducted to compare climate among the sampled regions (Tunisia was excluded due to the small samples and its climatic similarity to Morocco) and to analyze potential relationships among regional dominance of parasitoid species and bioclimatic variables in the three SubSaharan countries. A set of eight bioclimatic variables were selected for the analyses: annual mean temperature (Ann tem), maximum temperature of the warmest month (Max tem), minimum temperature of the coldest month (Min tem), mean temperature of the warmest quarter (Warm tem), mean temperature of the coldest quarter (Cold tem), annual precipitation (Ann prec), precipitation of the wettest quarter (Wet prec) and precipitation of the driest quarter (Dry prec). These bioclimatic variables were extracted from the WorldClim Global Climate Database 1.3 (http://www. worldclim.org) using the R 3.1.3 release. These variables are 
considered biologically relevant and used commonly in species distribution studies. A biplot analysis was conducted to characterize the relationships.

For the analyses of seasonal host-parasitoid dynamics in South Africa, host fly density was estimated as the number of fly puparia per fruit. Because wild fruit is smaller than cultivated fruit, each wild fruit supports fewer flies, commonly one fly larvae per fruit ${ }^{43}$; therefore the fly density per fruit approximately matches the percentage of infested fruit (i.e. fruit infestation rate). Data were pooled from different sites to estimate monthly mean host density or fruit infestation rate, total parasitism by all braconid parasitoids, parasitoid diversity, and the relative abundance of each braconid parasitoids on unripe and ripe fruit, respectively. Mixed models were used to analyze the effects of fruit maturity (unripe vs. ripe) and seasonal climate (both were fixed effects) as well as year (random effect) on monthly host density and total parasitism. Monthly mean temperature was used to represent a seasonal climate variable as precipitation was considered similar within the surveyed areas and other temperature parameters (e.g. maximum or minimum temperature) are highly correlated with the mean temperature. The temperature data were obtained from Weather Information (https://us.worldweatheronline.com/) from the closest cities (Stellenbosch, Paarl, Citrusdal, Cape Town, Bonnievale or Wellington) of the sampled sites. Generalized linear models (GLM) were applied to analyze the effects of (1) fruit maturity, mean monthly temperature, host density and parasitism on diversity, and (2) fruit maturity, mean monthly temperature, host density, and incidence of other parasitoids on the relative abundance of two major parasitoids ( $U$. africanus and $P$. lounsburyi). For GLM analyses, fruit maturity was coded categorically as 1 and 2 for unripe and ripe fruit, respectively, and parasitoid species incidence was coded as 1 (present) and 0 (absent). Percentage data were modelled with binomial distribution and a logit link function while the diversity data was modeled with Poisson distribution and a log link function. Statistical analyses were performed using JMP Pro ver13 (SAS 2013, Cary, NC).

\section{Declarations}

\section{Acknowledgements}

We thank Robert Copeland and Peris Machera for help with parasitoid collections in Kenya, and Rob Stotter and Jeanne de Waal with parasitoid collections, processing and shipping of materials at Stellenbosch University; John Andrews, John Hutchins and Karmit Levi for help rearing parasitoid material in Quarantines at UC Berkeley and Nassera Kadiri at EBCL from African shipments; Bob Wharton (retired, Texas A\&M university) for parasitoid species identification, Richard Stouthamer (University of California, Riverside), Marie Claude Bon (EBCL) and Mathew Middleton (formerly University of California, Berkeley) for confirming parasitoid populations through molecular analyses. Funds were provided by the California Specialty Crop Block Grant, California Olive Committee, USDA APHIS, CDFA Biological Control Program, and the USDA-CSREES Special Grants Program: Pest Management Alternatives.

Author contribution: XW, KD and RS analyzed data and wrote the initial manuscript draft. VW, KH, AK and CP conducted collections of parasitoids in Africa; KD, KH and $\mathrm{CP}$ secured funding. All authors contributed to the editing of the manuscript.

\section{Competing interests:}


The authors declare no competing interests.

\section{Additional information}

Data available from the Dryad Digital Repository (to be added)

Supplementary information is available for this paper (to be added)

Correspondence and requests for materials should be addressed to K.M.D. and X.G.W.

Reprints and permissions information is available at www.nature.com/reprints.

Publisher's note Springer Nature remains neutral with regard to jurisdictional claims in published maps and institutional affiliations.

\section{References}

1 Wolfe, L. M. Why alien invaders succeed: Support for the escape-from-enemy hypothesis. Am Nat 160, 705711, doi:10.1086/343872 (2002).

2 Facon, B. et al. A general eco-evolutionary framework for understanding bioinvasions. Trends Ecol Evol 21, 130-135, doi:10.1016/j.tree.2005.10.012 (2006).

3 Van Driesche, R. G. et al. Classical biological control for the protection of natural ecosystems. Biol Control 54, S2-S33, doi:10.1016/j.biocontrol.2010.03.003 (2010).

4 Hajek, A. E. et al. Exotic biological control agents: A solution or contribution to arthropod invasions? Biol Invasions 18, 953-969, doi:10.1007/s10530-016-1075-8 (2016).

5 Schwarzlander, M., Hinz, H. L., Winston, R. L. \& Day, M. D. Biological control of weeds: an analysis of introductions, rates of establishment and estimates of success, worldwide. Biocontro/ 63, 319-331, doi:10.1007/s10526-018-9890-8 (2018).

6 Naranjo, S. E., Ellsworth, P. C. \& Frisvold, G. B. Economic value of biological control in integrated pest management of managed plant systems. Annu Rev Entomo/60, 621-645, doi:10.1146/annurev-ento-010814021005 (2015).

7 Hoddle, M. S., Lake, E. C., Minteer, C. R. \& Daane, K. M. in Biological Control: A Global Initiative (eds P. G. Mason \& N. Dennis) (CSIRO Publishing, 2020).

8 Heimpel, G. E. \& Cock, M. J. W. Shifting paradigms in the history of classical biological control. Biocontrol 63, 27-37, doi:10.1007/s10526-017-9841-9 (2018).

9 Hoelmer, K. A. \& Kirk, A. A. Selecting arthropod biological control agents against arthropod pests: can the science be improved to decrease the risk of releasing ineffective agents? Biol Contro/34, 255-264, doi:10.1016/j.biocontrol.2005.05.001 (2005). 
10 Wang, X. G., Johnson, M. W., Daane, K. M. \& Yokoyama, V. Y. Larger olive fruit size reduces the efficiency of Psyttalia concolor, as a parasitoid of the olive fruit fly. Biol Control 49, 45-51, doi:10.1016/j.biocontrol.2009.01.004 (2009).

11 Sivinski, J., Pinero, J. \& Aluja, M. The distributions of parasitoids (Hymenoptera) of Anastrepha fruit flies (Diptera: Tephritidae) along an altitudinal gradient in Veracruz, Mexico. Biol Control 18, 258-269 (2000).

12 Wharton, R. A. in Fruit flies: Their Biology, Natural Enemies and Control Vol. 3B (eds A. S. Robinson \& G. Hooper) 303-313 (Elsevier, 1989).

13 Purcell, M. F. Contribution of biological control to integrated pest management of tephritid fruit flies in the tropics and subtropics. Integr Pest Manage Rev 3, 63-83 (1998).

14 Ovruski, S. M., Aluja, M., Sivinski, J. \& Wharton, R. A. Hymenopteran parasitoids on fruit-infesting Tephritidae (Diptera) in Latin America and the southern United States: diversity, distribution, taxonomic status and their use in fruit fly biological control. Integr Pest Manage Rev 5, 81-107 (2000).

15 Mohamed, S. A., Ramadan, M. M. \& Ekesi, S. in Fruit Fly Research and Development in Africa - Towards a Sustainable Management Strategy to Improve Horticulture (eds S. Ekesi, S. Mohamed, \& M. De Meyer) 325368 (Springer International Publishing, Cham., 2006).

16 Garcia, F. R. M., Ovruski, S. M., Suarez, L., Cancino, J. \& Liburd, O. E. Biological control of tephritid fruit flies in the Americas and Hawaii: A review of the use of parasitoids and predators. Insects 11, doi:10.3390/insects11100662 (2020).

17 Wharton, R. A. \& Yoder, M. J. Wharton RA, Yoder MJ. 2017. Parasitoids of fruit-infesting tephritidae. http://paroffit.org. Accessed on November 15, 2020. (2017).

18 Daane, K. M. \& Johnson, M. W. Olive fruit fly: Managing an ancient pest in modern times. Annu Rev Entomo/ 55, 155-169, doi:10.1146/annurev.ento.54.110807.090553 (2010).

19 Tzanakakis, M. E. Seasonal development and dormancy of insects and mites feeding on olive: A review. Neth J Zool 52, 87-224 (2003).

20 Green, P. S. A revision of Olea L. (Oleaceae). Kew Bull 57, 91-140 (2002).

21 Bon, M. C. et al. Populations of Bactrocera oleae (Diptera: Tephritidae) and its parasitoids in Himalayan Asia. Ann Entomol Soc Am 109, 81-91, doi:10.1093/aesa/sav114 (2016).

22 Zygouridis, N. E., Augustinos, A. A., Zalom, F. G. \& Mathiopoulos, K. D. Analysis of olive fly invasion in California based on microsatellite markers. Heredity 102, 402-412 (2009).

23 Augustinos, A. A. et al. Microsatellite analysis of olive fly populations in the Mediterranean indicates a westward expansion of the species. Genetica 125, 231-241 (2005).

24 Nardi, F. et al. Domestication of olive fly through a multi-regional host shift to cultivated olives: Comparative dating using complete mitochondrial genomes. Mol Phylogenet Evo/ 57, 678-686, 
25 Neuenschwander, P., Bigler, F., Delucchi, V. \& Michelakis, S. E. Natural enemies of preimaginal stages of Dacus oleae Gmel. (Dipt., Tephritidae) in Western Crete. I. Bionomics and phenologies. Boll Lab Entomol Agrar Filippo Silvestri 40, 3-32 (1983).

26 Boccaccio, L. \& Petacchi, R. Landscape effects on the complex of Bactrocera oleae parasitoids and implications for conservation biological control. BioControl 54, 607-616, doi:10.1007/s10526-009-9214-0 (2009).

27 Kapaun, T., Nadel, H., Headrick, D. \& Vredevoe, L. Biology and parasitism rates of Pteromalus nr. myopitae (Hymenoptera: Pteromalidae), a newly discovered parasitoid of olive fruit fly Bactrocera oleae (Diptera: Tephritidae) in coastal California. Biol Contro/ 53, 76-85, doi:10.1016/j.biocontrol.2009.11.002 (2010).

28 Silvestri, F. Report on an expedition to Africa in search of natural enemies of fruit flies (Trupaneidae) with descriptions, observations and biological notes. Hawaii Board of Agriculture and Forestry, Division of Entomology Bulletin 3, 1-146 (1914).

29 Hoelmer, K. A., Kirk, A. A., Pickett, C. H., Daane, K. M. \& Johnson, M. W. Prospects for improving biological control of olive fruit fly, Bactrocera oleae (Diptera: Tephritidae), with introduced parasitoids (Hymenoptera). Biocontrol Sci Technol 21, 1005-1025, doi:10.1080/09583157.2011.594951 (2011).

30 Greathead, D. J. \& Greathead, A. H. Biological control of insect pests by insect parasitoids and predators: the BIOCAT database. Biocontrol News and Information 13, 61N-68N (1992).

31 Neuenschwander, P. Searching parasitoids of Dacus oleae (Gmel) (Dipt., Tephritidae) in South Africa. J Appl Entomol 94, 509-522 (1982).

32 Loni, A. Developmental rate of Opius concolor (Hym.: Braconidae) at various constant temperatures. Entomophaga 42, 359-366 (1997).

33 Miranda, M. A., Miquel, M., Terrassa, J., Melis, N. \& Monerris, M. Parasitism of Bactrocera oleae(Diptera, Tephritidae) by Psyttalia concolor (Hymenoptera, Braconidae) in the Balearic Islands (Spain). J Appl Entomol 132, 798-805 (2008).

34 Muller, F. A., Dias, N. P., Gottschalk, M. S., Garcia, F. R. M. \& Nava, D. E. Potential distribution of Bactrocera oleae and the parasitoids Fopius arisanus and Psyttalia concolor, aiming at classical biological control. Biol Control 132, 144-151, doi:10.1016/j.biocontrol.2019.02.014 (2019).

35 Chardonnet, F., Blanchet, A., Hurtrel, B., Marini, F. \& Smith, L. Mass-rearing optimization of the parasitoid Psyttalia lounsburyi for biological control of the olive fruit fly. J App/ Entomol 143, 277-288, doi:10.1111/jen.12573 (2019).

36 La-Spina, M. et al. Effect of exposure time on mass-rearing production of the olive fruit fly parasitoid, Psyttalia lounsburyi (Hymenoptera: Braconidae). J Appl Entomol 142, 319-326, doi:10.1111/jen.12478 (2018). 
37 Daane, K. M. et al. Classic biological control of olive fruit fly in California, USA: release and recovery of introduced parasitoids. BioContro/ 60, 317-330, doi:10.1007/s10526-015-9652-9 (2015).

38 Wharton, R. A. \& Gilstrap, F. Key to and status of opiine braconid (Hymenoptera) parasitoids used in biological control of Ceratitis and Dacus s.l. (Diptera: Tephritidae). Ann Entomol Soc Am 76, 721-742 (1983).

39 Sime, K. R. et al. Psyttalia ponerophaga (Hymenoptera: Braconidae) as a potential biological control agent of olive fruit fly Bactrocera oleae (Diptera: Tephritidae) in California. Bull Entomol Res 97, 233-242, doi:10.1017/S0007485307004865 (2007).

40 Sime, K. R. et al. The biology of Bracon celer as a parasitoid of the olive fruit fly. BioControl 51, 553-567, doi:10.1007/s10526-005-6079-8 (2006).

41 Sime, K. R. et al. Diachasmimorpha longicaudata and D. kraussii (Hymenoptera: Braconidae), potential parasitoids of the olive fruit fly. Biocontrol Sci Technol 16, 169-179, doi:10.1080/09583150500188445 (2006).

42 Sime, K. R., Daane, K. M., Messing, R. H. \& Johnson, M. W. Comparison of two laboratory cultures of Psyttalia concolor (Hymenoptera: Braconidae), as a parasitoid of the olive fruit fly. Biol Contro/ 39, 248-255, doi:10.1016/j.biocontrol.2006.06.007 (2006).

43 Mkize, N., Hoelmer, K. A. \& Villet, M. H. A survey of fruit-feeding insects and their parasitoids occurring on wild olives, Olea europaea ssp cuspidata, in the Eastern Cape of South Africa. Biocontrol Sci Technol 18, 9911004 (2008).

44 Sime, K. R., Daane, K. M., Wang, X.-G., Johnson, M. W. \& Messing, R. H. Evaluation of Fopius arisanus as a biological control agent for the olive fruit fly in California. Agricul Forest Entomol 10, 423-431, doi:10.1111/j.1461-9563.2008.00401.x (2008).

45 Wang, X. G. \& Messing, R. H. Potential interactions between pupal and egg- or larval-pupal parasitoids of tephritid fruit flies. Environ Entomo/ 33, 1313-1320 (2004).

46 Wang, X. G. \& Messing, R. H. The ectoparasitic pupal parasitoid, Pachycrepoideus vindemmiae (Hymenoptera: Pteromalidae), attacks other primary tephritid fruit fly parasitoids: host expansion and potential non-target impact. Biol Control 31, 227-236 (2004).

47 Wang, X. G., Levy, K., Son, Y., Johnson, M. W. \& Daane, K. M. Comparison of the thermal performance between a population of the olive fruit fly and its co-adapted parasitoids. Biol Control 60, 247-254, doi:10.1016/j.biocontrol.2011.11.012 (2012).

48 Wang, X.-G., Johnson, M. W., Yokoyama, V. Y., Pickett, C. H. \& Daane, K. M. Comparative evaluation of two olive fruit fly parasitoids under varying abiotic conditions. Biocontro/ 56, 283-293, doi:10.1007/s10526-0109332-8 (2011).

49 Daane, K. M. et al. Biological control of the olive fruit fly in California. Calif Agric 65, 21-28 (2011). 
50 Wang, X. G. et al. Crop domestication relaxes both top-down and bottom-up effects on a specialist herbivore. Basic Appl Ecol 10, 216-227, doi:10.1016/j.baae.2008.06.003 (2009).

51 Nadel, H., Daane, K. M., Hoelmer, K. A., Pickett, C. H. \& Johnson, M. W. Non-target host risk assessment of the idiobiont parasitoid, Bracon celer (Hymenoptera: Braconidae), for biological control of olive fruit fly in California. Biocontrol Sci Technol 19, 701-715, doi:10.1080/09583150902974384 (2009).

52 Wharton, R. A. et al. Parasitoids of medfly, Ceratitis capitata, and related tephritids in Kenyan coffee: a predominantly koinobiont assemblage. Bull Entomol Res 90, 517-526 (2000).

53 Kimani-Njogu, S. W., Trostle, M. K., Wharton, R. A., Woolley, J. B. \& Raspi, A. Biosystematics of the Psyttalia concolor species complex (Hymenoptera: Braconidae: Opiinae): the identity of populations attacking Ceratitis capitata (Diptera: Tephritidae) in coffee in Kenya. Biol Control 20, 167-174 (2001).

54 Rugman-Jones, P. F., Wharton, R., van Noort, T. \& Stouthamer, R. Molecular differentiation of the Psyttalia concolor (Szépligeti) species complex (Hymenoptera: Braconidae) associated with olive fly, Bactrocera oleae (Rossi) (Diptera: Tephritidae), in Africa. Biol Control 49, 17-26, doi:10.1016/j.biocontrol.2008.12.005 (2009).

55 Billah, M. K. et al. Cross mating studies among five fruit fly parasitoid populations: potential biological control implications for tephritid pests. BioControl 53, 709-724 (2008).

56 Narayanan, E. S. \& Chawla, S. S. Parasites of fruit fly pests of the world. Beitrage zur Entomologie 12, 437476 (1962).

57 Neuenschwander, P. Searching parasitoids of Dacus oleae in South Africa. Zeitschrift fur Angewandte Entomologie 94, 509-522 (1982).

58 Daane, K. M. et al. Psyttalia lounsburyi (Hymenoptera: Braconidae), potential biological control agent for the olive fruit fly in California. Biol Control 44, 78-89, doi:10.1016/j.biocontrol.2007.08.010 (2008).

59 Benelli, G. et al. Behavioral and electrophysiological responses of the parasitic wasp Psyttalia concolor (Szepligeti) (Hymenoptera: Braconidae) to Ceratitis capitata-induced fruit volatiles. Biol Control 64, 116-124, doi:10.1016/j.biocontrol.2012.10.010 (2013).

60 Raspi, A. \& Loni, A. Alcune note sull'allevamento massale di Opius concolor Szépligeti (Hym.: Braconidae) e su recnti tentative d'introduzione della specie in Toscana e Liguria. Frustula Entomologia 30, 135-145 (1994).

61 Yokoyama, V. Y. et al. Performance of Psyttalia humilis (Hymenoptera: Braconidae) reared from irradiated host on olive fruit fly ( Diptera: Tephritidae) in California. Environ Entomol 41, 497-507, doi:10.1603/en11252 (2012).

62 Yokoyama, V. Y. et al. Response of Psyttalia cf. concolor to olive fruit fly (Diptera: Tephritidae), high temperature, food, and bait sprays in California. Environ Entomo/ 40, 315-323 (2010).

63 Yokoyama, V. Y. et al. Field performance and fitness of an olive fruit fly parasitoid, Psyttalia humilis (Hymenoptera: Braconidae), mass reared on irradiated Medfly. Biol Control 54, 90-99, 
doi:10.1016/j.biocontrol.2010.04.008 (2010).

64 Wang, X. G. et al. Overwintering survival of olive fruit Fly (Diptera: Tephritidae) and two introduced parasitoids in California. Environ Entomol 42, 467-476, doi:10.1603/en12299 (2013).

65 Daane, K. M., Wang, X. G., Johnson, M. W. \& Cooper, M. L. Low temperature storage effects on two olive fruit fly parasitoids. BioContro/ 58, 175-185, doi:10.1007/s10526-012-9481-z (2013).

66 Johnson, M. W. et al. High temperature impacts olive fruit fly population dynamics in California's Central Valley. Calif Agric 65, 29-33 (2011).

\section{Figures}
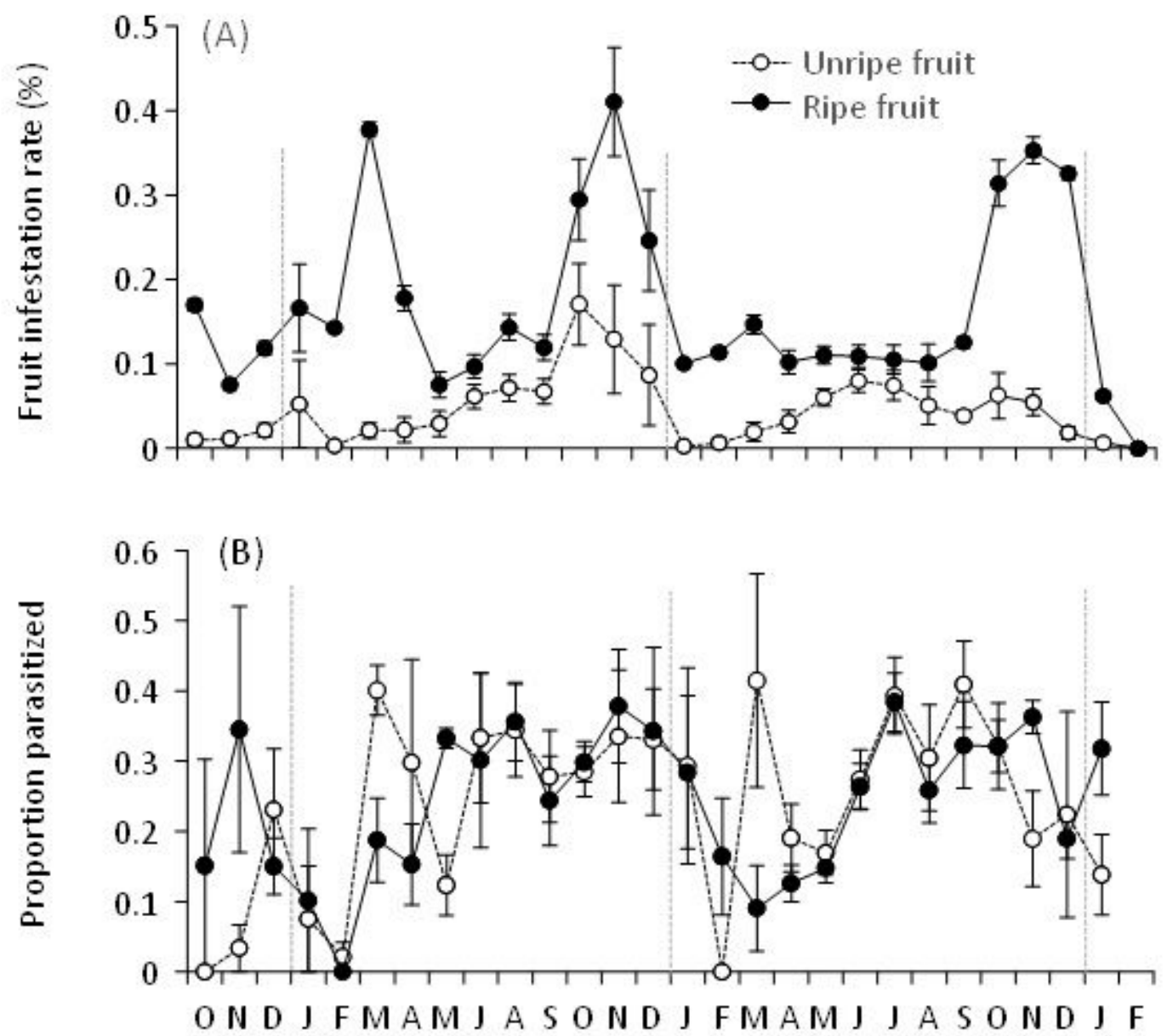

Month of year (October 2004 to February 2007)

\section{Figure 1}

For both unripe and ripe fruit, the seasonal dynamics of (A) combined host density of B. oleae and B. biguttula and (B) parasitism by braconid parasitoids from October 2004 to February 2007 in the Western Cape, South Africa. Values are mean and SE and data were pooled from different collection sites. 


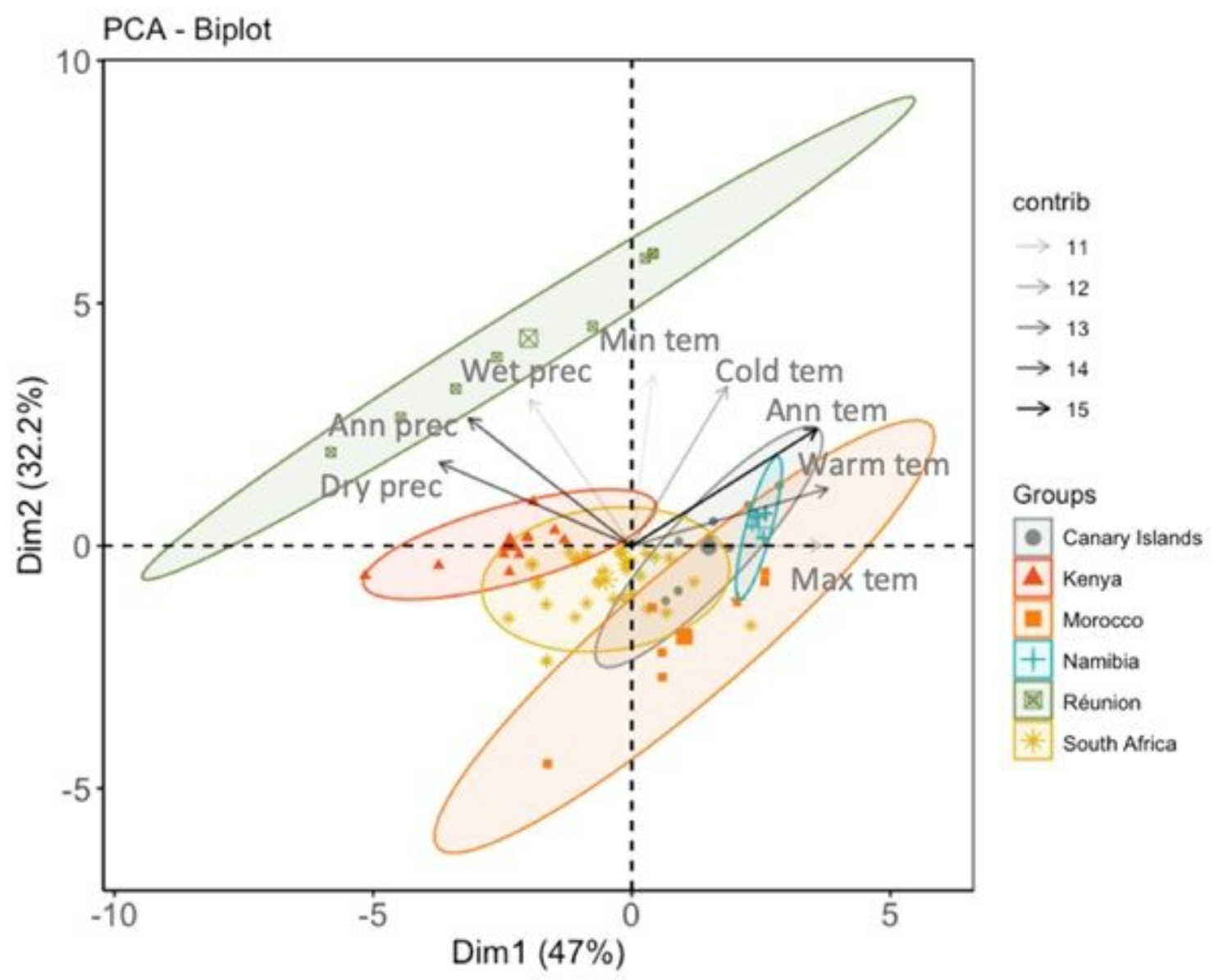

Figure 1

Principal Component Analysis ordination of explored regions based on the two principal climatic gradients. The enlarged symbols indicate the centroids of the inertia ellipses while arrows indicate the importance of each bioclimatic variable on the two significant components. Climatic predictors are: Ann tem = annual mean temperature, Max tem = maximum temperature of the warmest month, Min tem = minimum temperature of the coldest month, Warm tem $=$ mean temperature of the warmest quarter, Cold tem = mean temperature of the coldest quarter, Ann prec $=$ annual precipitation, Wet prec $=$ precipitation of the wettest quarter, and Dry prec $=$ precipitation of the driest quarter. 

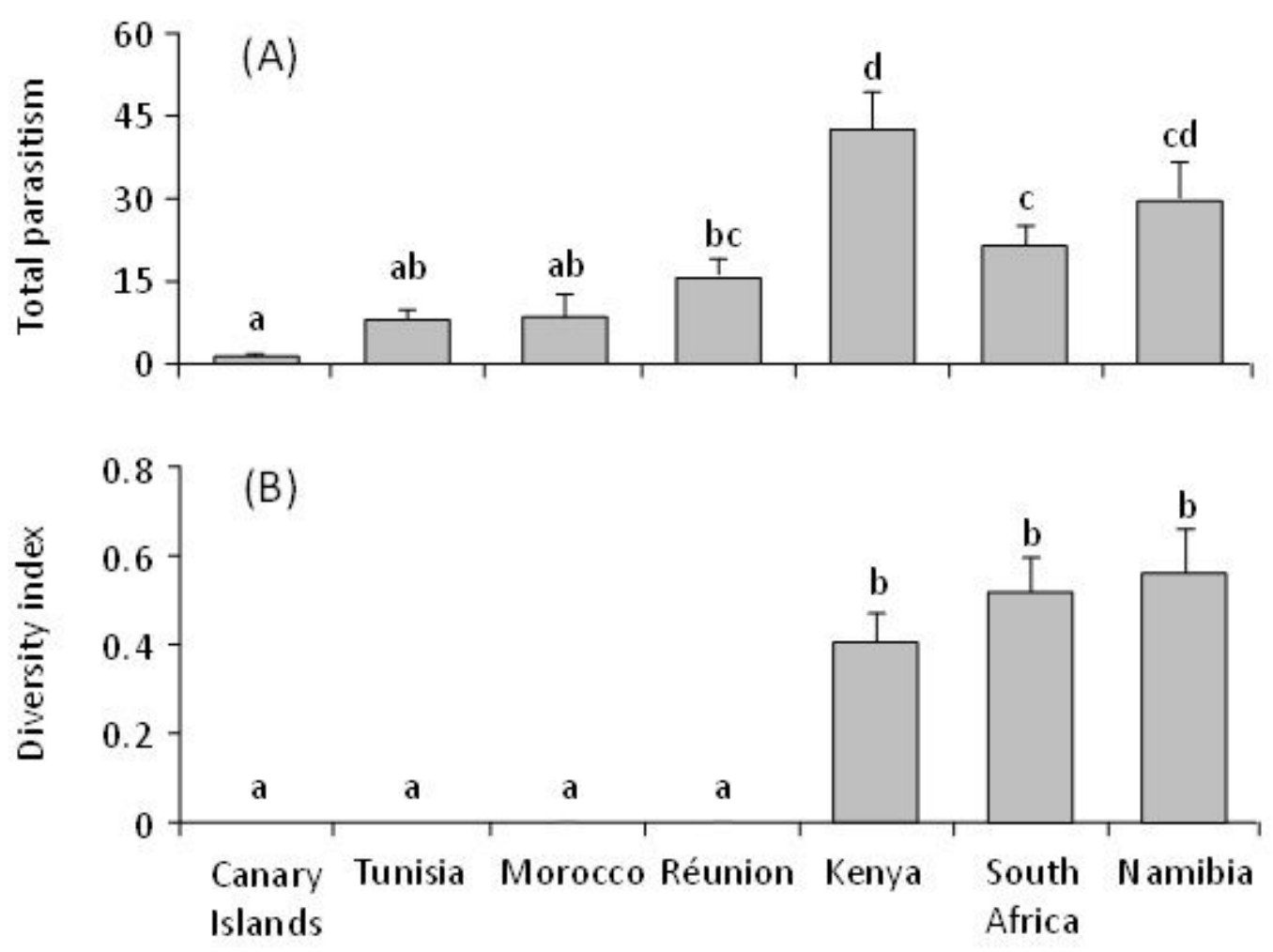

Figure 1

(A) Percentage parasitism of Bactrocera spp. (emerged parasitoids / (emerged parasitoids + flies)) and (B) parasitoid species diversity (Shannon index) of the braconid parasitoid species reared from the collected flies in different regions in Africa; bars refer to mean and SE and different letters above the standard error bars indicate significant differences $(P<0.05)$. 


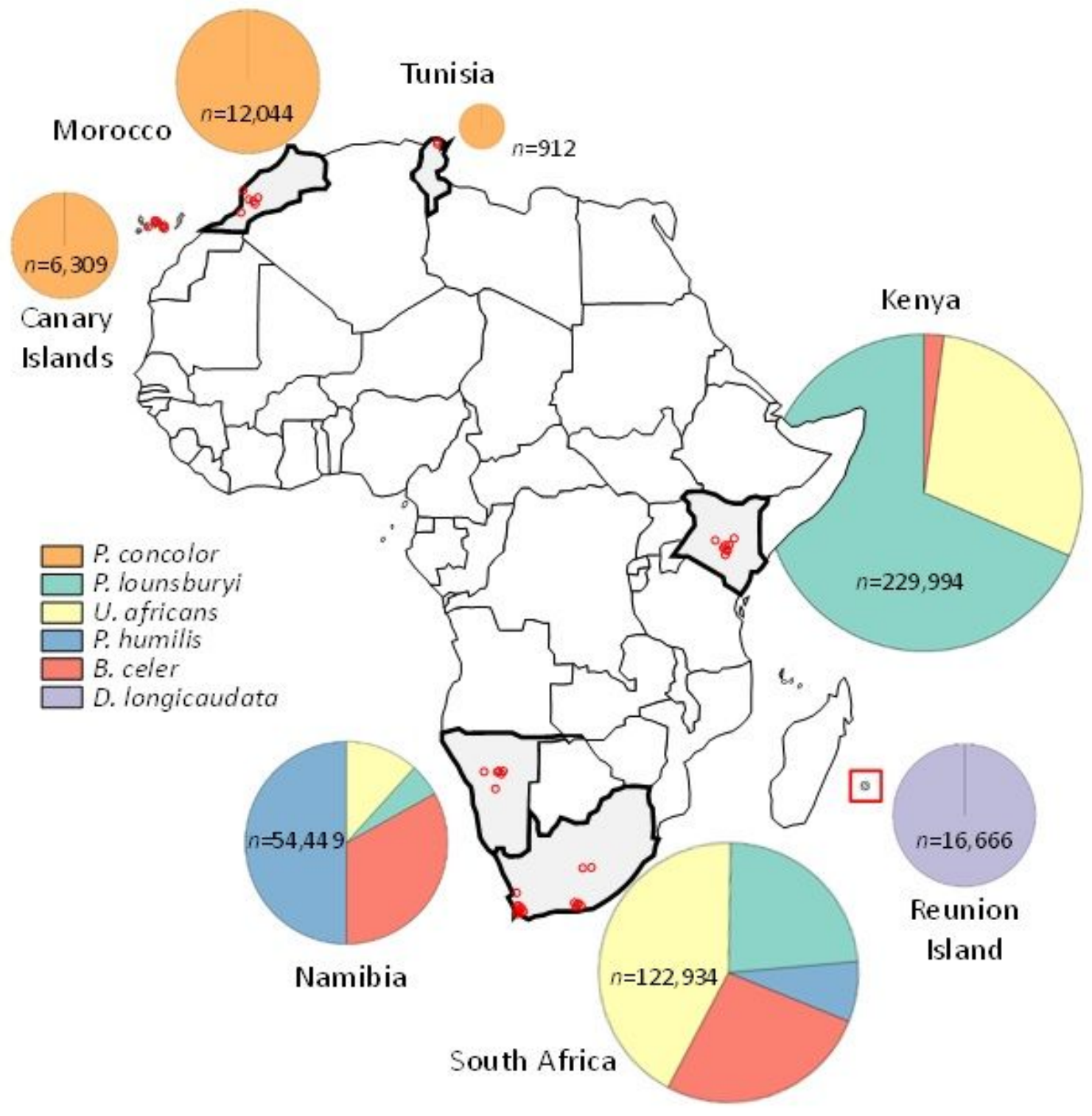

Figure 1

Composition and relative abundance (\%) of braconid parasitoid species reared from Bactrocera spp. on wild olives in seven regions of Africa; small red circles show approximate locations of sampling sites and the parasitoid composition size is proportional to the number of fruits $(n)$ collected in each region. 
(A)
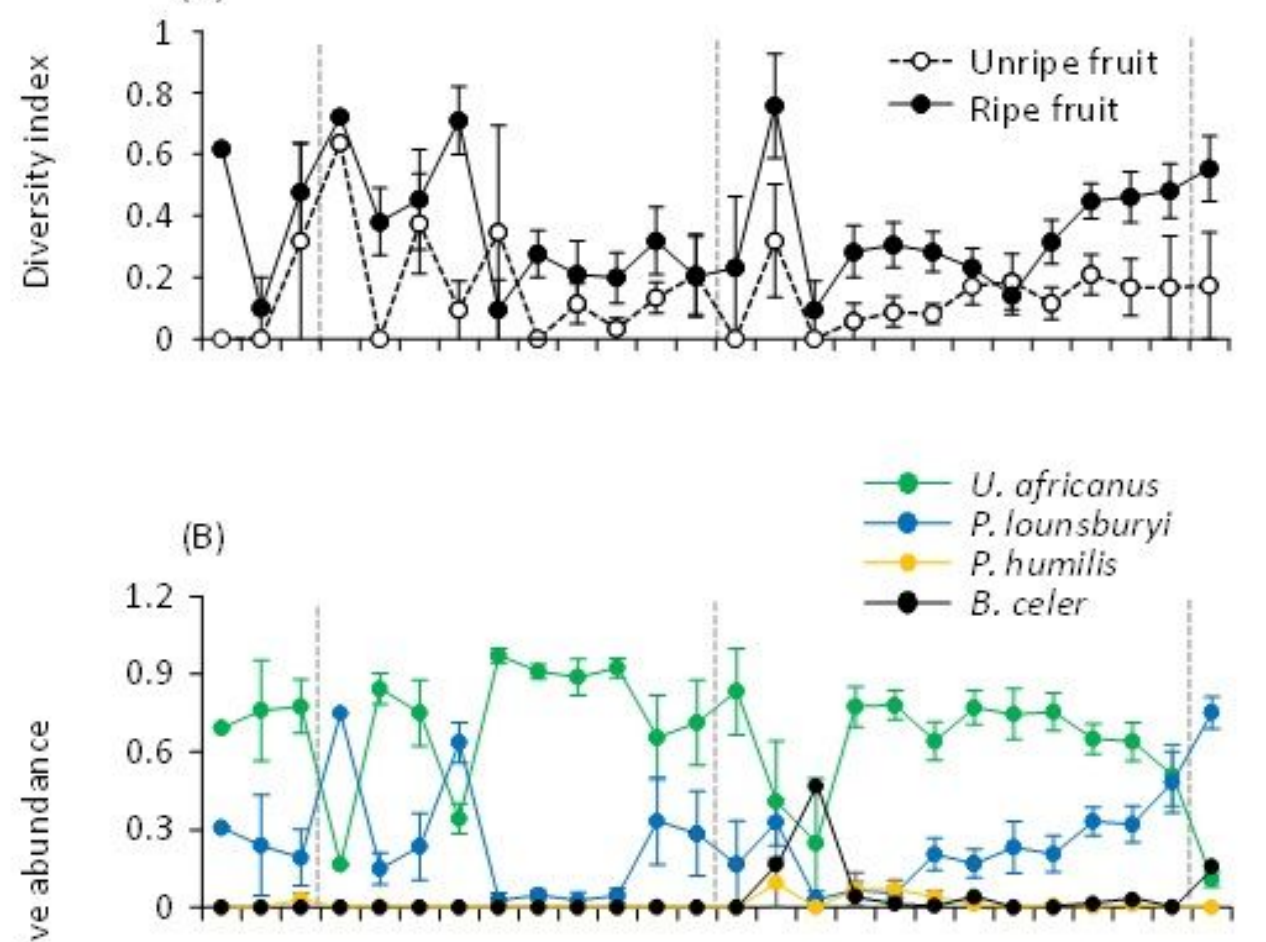

(C)

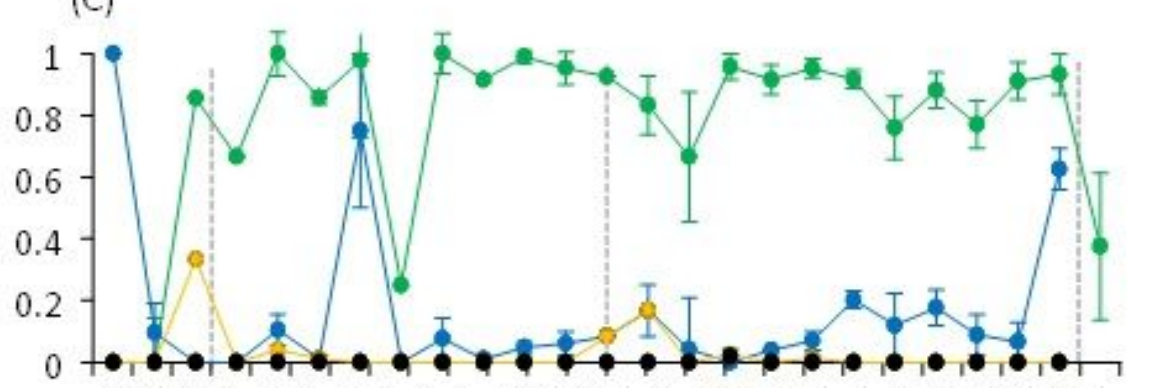

ONDJMAMJASONDJFMAMJ J ASONDJ

Mon th of year (October 2004 to February 2007)

\section{Figure 1}

For both unripe and ripe fruit, the seasonal dynamics of (A) parasitoid species diversity (Shannon index) of the braconid parasitoid species reared from the collected flies, and relative abundance of braconid parasitoid species reared from $B$. oleae and B. biguttula on (B) ripe fruit and (C) unripe fruit in the Western Cape, South Africa. Values are mean and SE and data were pooled from different collection sites. 


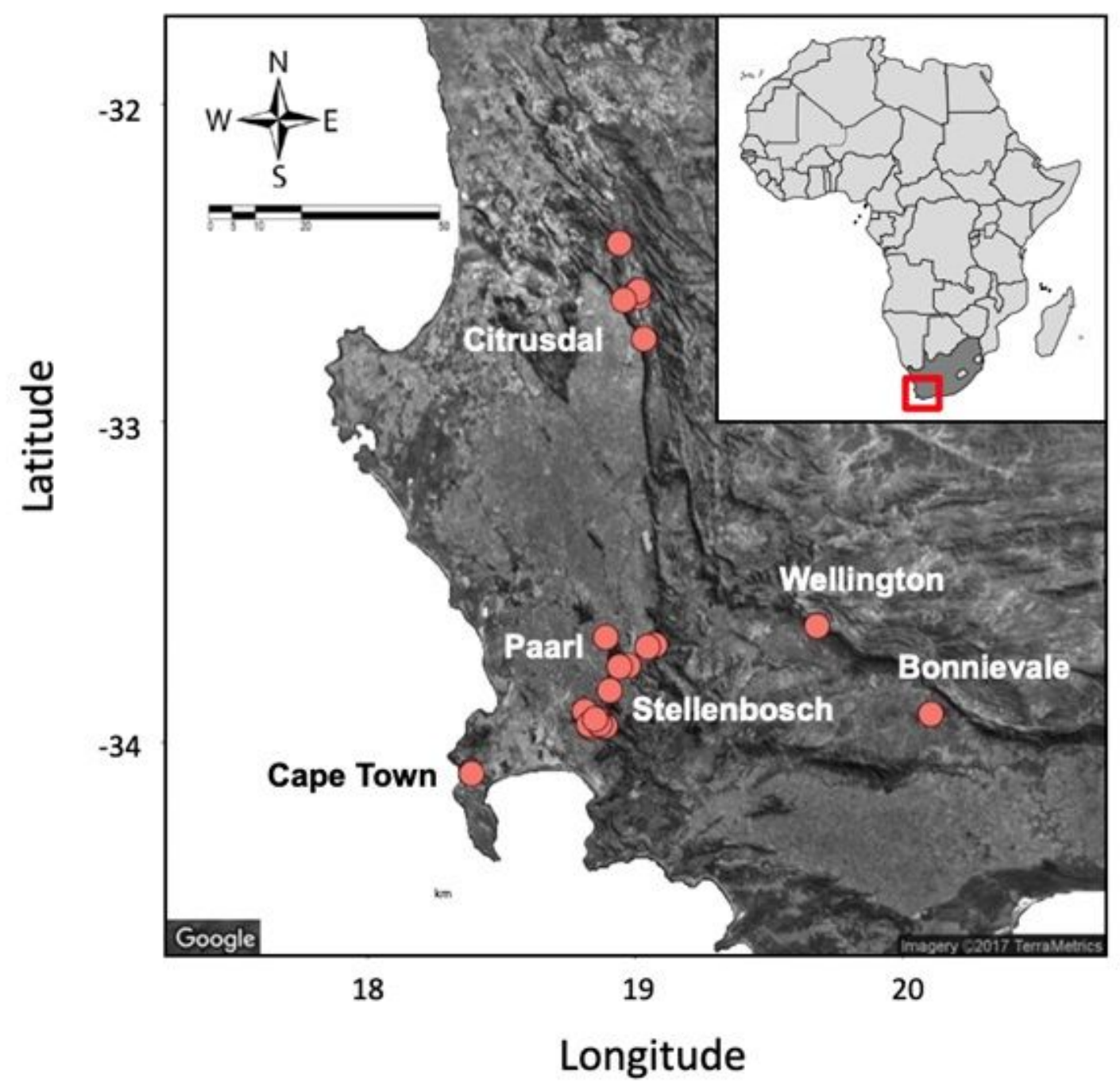

Figure 1

Sampled sites where the seasonal dynamics of Bactrocera spp. and their parasitoids were monitored (20042007) on wild olives in the Western Cape, South Africa. 


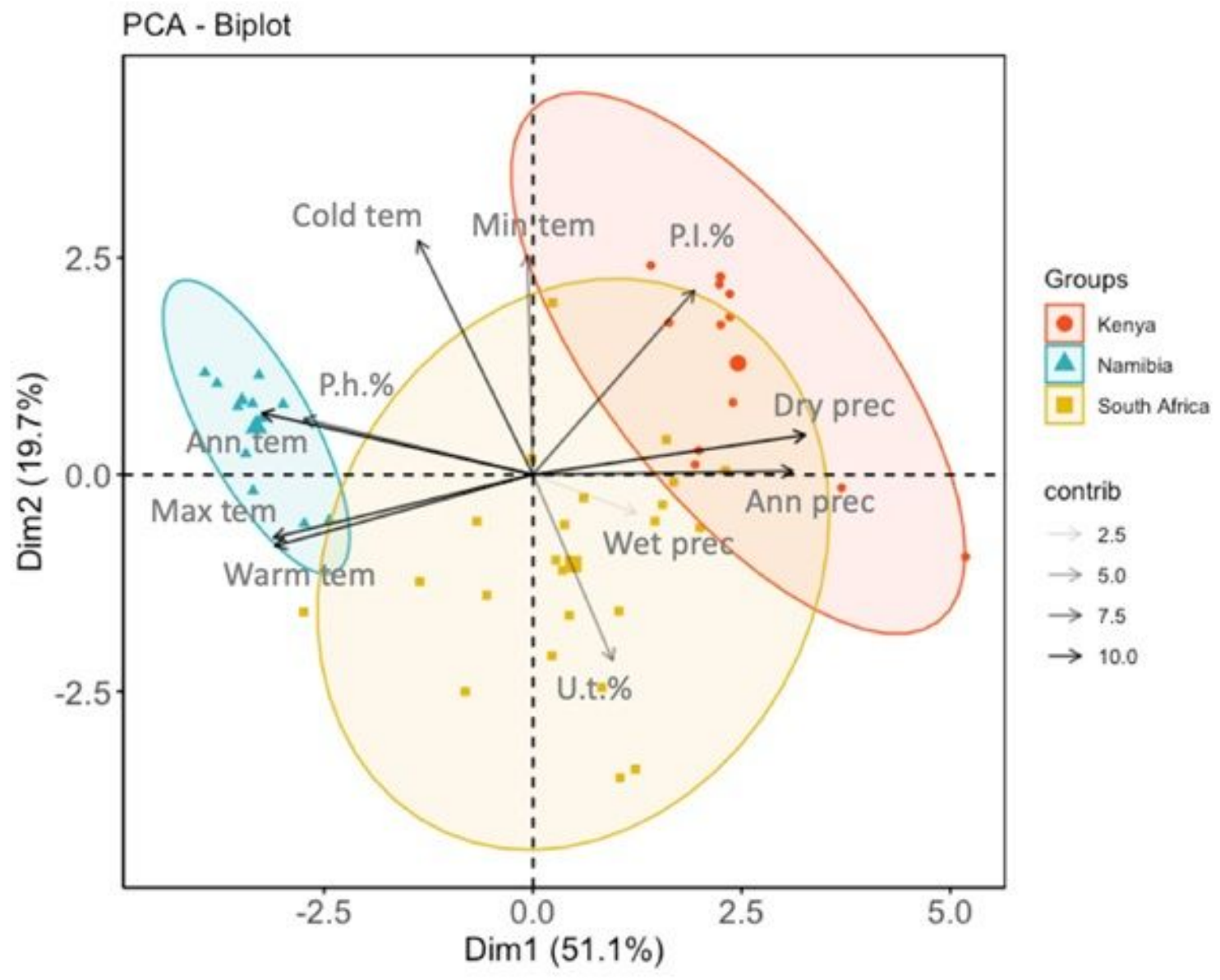

Figure 1

Principal Component Analysis ordination of sampling sites in three Sub-Saharan countries based on the relative abundance of the dominant parasitoid species (P. lounsburyi, P. humilis and U. africanus). The biplot shows the relationships between bioclimatic variables and dominance of parasitoid species in each region. Climatic predictors are: Ann tem = annual mean temperature, Max tem = maximum temperature of the warmest month, Min tem = minimum temperature of the coldest month, Warm tem = mean temperature of the warmest quarter, Cold tem $=$ mean temperature of the coldest quarter, Ann prec $=$ annual precipitation, Wet prec $=$ precipitation of the wettest quarter, Dry prec $=$ precipitation of the driest quarter, and the relative proportion of $\mathrm{P}$. lounsburyi (P.I.\%), P. humilis (P.h.\%) and U. africanus (U.t.\%) to emerge from parasitized fruits.

\section{Supplementary Files}

This is a list of supplementary files associated with this preprint. Click to download.

- SupplementalFigure1.docx 